\title{
Belphégor
}

\section{Alice Favaro e Irene Incarico (dir.), Eurofumetto \& Globalizzazione. Studi su graphic novel e linguaggi dei comics}

\section{Paolo Matteucci}

\section{OpenEdition}

\section{Journals}

Electronic version

URL: https://journals.openedition.org/belphegor/1513

DOI: 10.4000/belphegor.1513

ISSN: 1499-7185

Publisher

LPCM

Electronic reference

Paolo Matteucci, "Alice Favaro e Irene Incarico (dir.), Eurofumetto \& Globalizzazione. Studi su graphic novel e linguaggi dei comics", Belphégor [Online], 17 | 2019, Online since 26 March 2019, connection on 25 August 2021. URL: http://journals.openedition.org/belphegor/1513 ; DOl: https://doi.org/10.4000/ belphegor.1513

This text was automatically generated on 25 August 2021

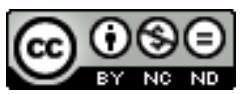

Belphégor est mis à disposition selon les termes de la Licence Creative Commons Attribution - Pas d'Utilisation Commerciale - Pas de Modification 4.0 International. 


\title{
Alice Favaro e Irene Incarico (dir.), Eurofumetto \& Globalizzazione. Studi su graphic novel e linguaggi dei comics
}

\author{
Paolo Matteucci
}

\section{REFERENCES}

Eurofumetto \& Globalizzazione. Studi su graphic novel e linguaggi dei comics. A cura di Alice Favaro e Irene Incarico. La Spezia : Cut-Up Publishing, 2018. 158 p. ISBN :

9788895246635 . 


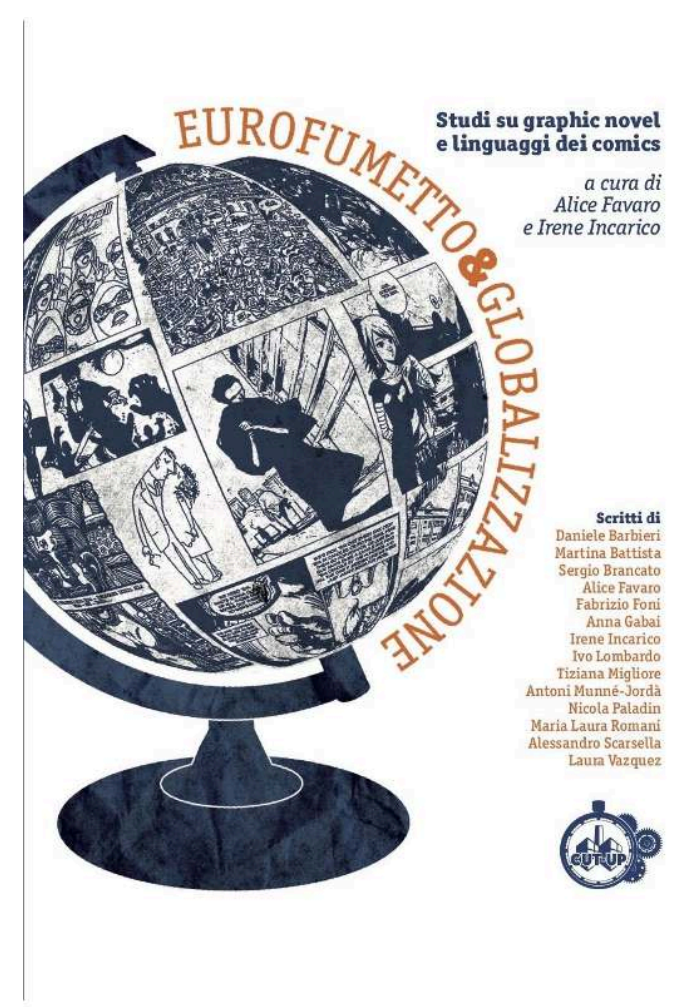

1 Eurofumetto \& Globalizzazione, realizzato sotto l'egida del Dipartimento di Studi Linguistici e Culturali Comparati dell'Università Ca' Foscari di Venezia e del Laboratorio per lo Studio Letterario del Fumetto (che promosse, nel 2014, tre giornate di studio sul tema che dà il titolo al volume), mira a sviluppare una serie di riflessioni per un'indagine trans-nazionale delle valenze storico-sociali, semantiche, tematiche, linguistiche e culturali del fumetto contemporaneo. Nel presentare al lettore una eterogenea raccolta di saggi accomunati dalla consapevolezza del fatto che il 'genere' fumetto, oltre a caratterizzarsi per la sua natura intrinsecamente poliedrica e proteiforme, rappresenta un costrutto mutevole e cangiante nello spazio e nel tempo, il volume non ha la pretesa di fornire uno studio esaustivo di tutte le modalità espressive attraverso cui il fumetto, anche nelle sue diverse "varianti terminologiche" (comics, sequential art, graphic novel, bande dessinée, eccetera), si è manifestato e si manifesta nella contemporaneità. Gli undici saggi inclusi nel libro, invece, si propongono-seppure non sempre esplicitamente-di interrogare la possibilità secondo cui, proprio a causa della sua natura metamorfica, il fumetto costituisca al tempo stesso "un linguaggio" ed un "mezzo di comunicazione" che, nel suo complesso e nelle sue complessità, si presenta ai lettori simultaneamente come "una forma d'intrattenimento" e "una forma d'arte".

2 Nel saggio intitolato “Fumetto europeo? È possibile definirlo?" Daniele Barbieri focalizza la propria attenzione sulle principali peculiarità (espressive, narrative, tecniche e contenutistiche) del fumetto francese, inglese, spagnolo ed italiano del Novecento. Dopo aver rilevato che il fumetto europeo ha incominciato ad assumere e sviluppare una propria fisionomia specifica solo quando, grossomodo a partire dal 1960, ha cominciato ad essere presentato al pubblico principalmente nelle vesti di un medium colto, politicamente impegnato, aperto ai temi dell'avventura, della fantascienza e dell'erotismo, Barbieri suggerisce di riconoscere il fatto che, nel contesto europeo, al di là delle peculiarità geopolitiche e linguistico-culturali che permeano la produzione 
fumettistica di ciascun paese, gli interscambi transnazionali sono significativi in misura superiore, o per lo meno uguale, alle diverse specificità e sensibilità locali.

3 Una riflessione sulle relazioni tra le trasformazioni del fumetto ed i più vasti cambiamenti occorsi, a partire dagli anni Settanta, nei sistemi mediatici di comunicazione è al centro del saggio, intitolato "Il fumetto e la post-serialità", di Sergio Brancato. Oltre a segnalare la mancanza di neutralità e altre complessità insite nell'adozione stessa della locuzione graphic novel (incluse certe valenze semantiche connesse alle nozioni di autorialità e serialità, che tra l'altro mettono in risalto $\mathrm{i}$ parallelismi tra lo sviluppo del cosiddetto romanzo grafico del secondo Novecento e la nascita del romanzo borghese moderno), l'estensore del saggio analizza la ridefinizione e la ricollocazione del graphic novel all'interno del processo di demassificazione delle pratiche contemporanee di comunicazione e, soprattutto, misura l'importanza della produzione e della divulgazione di fumetti all'interno degli odierni canali "immateriali" di trasmissione del sapere, a cominciare dal web.

4 Il saggio di Maria Laura Romani e Anna Gabai intitolato "Il fumetto nel mondo arabo" presenta, a beneficio del pubblico italofono, un'interessante introduzione alla storia ed alla diffusione del fumetto nei paesi di lingua araba. Nel loro intervento, le due autrici soffermano l'attenzione sulla produzione fumettistica egiziana e sviluppano un'analisi di tre rappresentativi case studies: il graphic novel "Metro" (2008) di Magdial-Shafi, l'antologia intitolata "Kharig al-saitara: Out of control" (2011) e la testata quadrimestrale nata nel 2010 "TokTok: la stazione delle storie illustrate", autofinanziata e autoprodotta da un collettivo di giovani artisti cairoti.

5 Nelle pagine successive Eurofumetto \& Globalizzazione presenta un excursus bibliografico, accompagnato da interessanti riproduzioni di tavole ed illustrazioni, relativo alla storia dei fumetti in lingua catalana, a firma di Antoni Munné-Jordà; un saggio in lingua spagnola, di Laura Vazquez, che rileva le continuità, non solo stilistiche ma anche discorsive, esistenti tra l'opera di Saul Steinberg (1914-1999) e quella di Copi (Raúl Damonte Botana, 1939-1987); un'indagine semiotica di stampo formalista, sviluppata da Tiziana Migliore, relativa alle relazioni tra pittura e fumetto e centrata sulle capacità, da parte di quest'ultimo, di inglobare e risemantizzare al suo interno, attraverso la scorniciatura, lo "statuto artistico" della cornice pittorica.

6 Il saggio più corposo di Eurofumetto \& Globalizzazione, corredato da un nutrito e intrigante apparato iconografico, si intitola "Mondo Tecno" e fornisce una succinta descrizione dell'opera-intrinsecamente distopica e controculturale, a larghi tratti lisergica e solo in parte riducibile al genere 'fumettistico'-dello spezzino Gianluca Lerici (1963-2006), meglio conosciuto dal pubblico con lo pseudonimo di Professor Bad Trip. L'intervento, sviluppato in maniera chiara e sintetica da Fabrizio Foni e Irene Incarico, ha innanzitutto il merito di iniziare a colmare, almeno in parte, le lacune dovute alla mancanza di studi accademici dedicati ad una delle figure più rappresentative della cultura underground italiana di fine Novecento. Nel corso del loro saggio, gli autori delineano i principali tratti caratteristici dell'opera grafica, illustrativa e fumettistica di Bad Trip; ne collocano puntualmente l'operato nella scena anarcopunk e nella galassia cyberpunk italiane; tracciano a grandi linee il complesso sistema di riferimenti, criptocitazioni, détournamenti e allusioni intertestuali che legano la produzione di Bad Trip alle opere-tra gli altri-di Hieronymus Bosch, Pieter Bruegel, Giuseppe Arcimboldo, Edvard Munch, William S. Burroughs e David 
Cronenberg; infine registrano il tardivo, e quasi interamente postumo, riconoscimento della creatività di Lerici da parte della cultura istituzionale.

7 I tre successivi interventi presentati da Eurofumetto \& Globalizzazione contengono, rispettivamente, una alquanto semplicistica serie di considerazioni relative alla circolazione di immagini, fumetti e informazioni sul web in epoca contemporanea; un sintetico sunto descrittivo, organizzato in ordine cronologico, dei principali fumetti seriali pubblicati da Sergio Bonelli Editore dal dopoguerra in poi; una breve analisi, redatta in lingua inglese, delle rappresentazioni della Prima Guerra Mondiale nelle fiction del fumetto statunitense degli anni Cinquanta e poi nel più recente graphic novel, sempre americano, intitolato "The Harlem Hellfighters" (2014).

Il volume si chiude con un interessante intervento, di Alice Favaro, in cui si sottolineano le potenzialità, da parte di fumetti e comics, di presentarsi quali mezzo privilegiato di denuncia, indagine ed informazione di fronte a gravi problemi sociali del passato e del presente. Oltre a fornire un'ampia serie di spunti interpretativi, questo saggio, intitolato "Nota per uno studio sulle forme di rappresentazione delle mutazioni sociali nel fumetto: diseguaglianza ed emigrazione", presenta una succinta analisi visivo-testuale di "Etenesh. L'odissea di una migrante" (2011) di Paolo Castoldi. A posteriori, il notevole potenziale euristico convogliato dall'intervento di Favaro sembra confermato dalla recente pubblicazione di alcuni lavori-come Cronache dalle periferie dell'impero di Antonella Selva, Salvezza di Marco Rizzo e Lelio Bonaccorso, e Un voyage sans retour di Gaspard Njock, tutti usciti nel 2018-apertamente dedicati a questioni sociali ed emergenze umanitarie, inclusi i flussi migratori che attraversano il Mediterraneo, di estrema attualità.

Eurofumetto \& Globalizzazione, nel suo complesso, raccoglie una serie di interventi di indubbio interesse, ciascuno dei quali permette agli "appassionati" così come agli "studiosi" (se si decide di approvare l'uso di tale dicotomia, cui peraltro fa esplicito riferimento la quarta di copertina) di identificare un certo numero di punti di partenza per lo sviluppo di ricerche future. In questa direzione non è di grande aiuto, nonostante il registro forbito e forse anche a causa della sua verbosità, la brevissima sezione introduttiva con cui si apre il volume. Al di là dei non pochi parallelismi e delle appariscenti connessioni tra i diversi interventi che, nel navigare il testo, il lettore è presumibilmente in grado di riconoscere da sé, Eurofumetto \& Globalizzazione non sembra infatti, nonostante il titolo, esplicitamente fondarsi su un riconoscibile quadro metodologico ed epistemologico d'insieme. Seppur ciascuno dei saggi fornisca, separatamente e individualmente, notevoli stimoli per la riflessione, il libro non è corredato da una riconoscibile e rigorosa cornice analitico-interpretativa che, per esempio, faccia emergere i legami (anche interdisciplinari) che connettono tra loro $\mathrm{i}$ diversi saggi oppure che, in maniera più generale, metta nitidamente a fuoco le complessità inscritte nella scelta di porre il fumetto a confronto del concettoanch'esso poliedrico, cangiante, spesso elusivo ed in ultima analisi proteiforme - di 'globalizzazione'. 


\section{AUTHOR}

PAOLO MATTEUCCI

Dalhousie University 